contribuera à l'activation de la p70-S6 kinase.

mTOR apparaît donc comme une cible pharmacologique de première importance dans le traitement de tumeurs dans lesquelles l'équilibre PI 3 kinase/PTEN est modifié en faveur de la PI 3-kinase. Un diagnostic moléculaire fondé sur l'analyse des mutations de PTEN ainsi que sur le niveau de phosphorylation de protéines impliquées dans la voie PI 3-kinase/PTEN (p70-S6 kinase ou $4 \varepsilon-B P 1$ par exemple) pourrait permettre d'identifier les patients susceptibles de répondre au $\mathrm{CCl}-779$ (actuellement en phase I d'essai clinique dans divers cancers). L'association du CCl-779 et d'un agent cytotoxique pourrait représenter une nouvelle approche dans le traitement de certains cancers $[3,4]$.

Au-delà de cet exemple, il apparaît aujourd'hui important de rechercher la dépendance potentielle d'une cellule cancéreuse pour une voie de

signalisation particulière afin de concevoir des inhibiteurs pharmacologiques les plus ciblés et les moins toxiques possibles. $\diamond$ The FRAP/mTOR kinase: a novel target for anticancer drugs?

1. Neshat MS, Mellinghoff IK, Tran C, et al. Enhanced sensitivity of PTENdeficient tumors to inhibition of FRAP/mTOR. Proc Natl Acad Sci USA 2001 ; 98 : 10314-9.

2. Podsypanina K, Lee RT, Politis C, et al. An inhibitor of $\mathrm{mTOR}$ reduces neoplasia and normalizes p70/S6 kinase activity in Pten ${ }^{+/}$ mice. Proc Natl Acad Sci USA 2001 ; 98 : 10320-5.

3. Mills GB, Lu Y, Kohn EC. Linking molecular therapeutics to molecular diagnostics : inhibition of the FRAP/RAFT/TOR component of the PI3K pathway preferentially blocks PTEN mutant cells in vitro and in vivo. Proc Natl Acad Sci USA 2001 ; 98 : 10031-3.

4. Brooksbank C. Therapeutic targets: withdrawal symptoms. Nat Rev Cancer 2001 ; 1 : 6-11.

5. Vanhaesebroeck $B$, Leevers S), Khatereh A, et al. Synthesis and function of 3-phosphorylated inositol lipids. Annu Rev Biochem 2001 ; 70 : 535-602.

6. Stein RC, Waterfield MD. PI 3-kinase inhibition : a target for drug development? Mol Med Today 2000 ; 6 : 347-57.

7. Payrastre B, Missy K, Giuriato $S$, et al. Phosphoinositides : key players in cell signalling, in time and space. Cell Signal $2001 ; 13: 377-87$.

8. Di Cristofano A, Pandolfi PP. The multiples roles of PTEN in tumor suppression. Cell $2000 ; 100$ : 387-90.

9. Ali IU, Schriml LM, Dean M. Mutational spectra of PTEN/MMAC gene : a tumor suppressor with lipid phosphatase activity. J Natl Cancer Inst 1999 ; 91 : 1922-32.

10. Maehama T, Taylor GS, Dixon JE. PTEN and myotubularin : novel phosphoinositide phosphatases. Annu Rev Biochem 2001 ; 70 : 247-79

\title{
NOUVELLE
}

\section{Le collagène de type XVIII, un nouveau collagène impliqué dans l'arborisation épithéliale rénale et pulmonaire}

Brigitte Lelongt, Pierre Ronco

$>$ La première étape du développement rénal est caractérisée par l'invasion d'un amas de cellules mésenchymateuses indifférenciées par une structure épithéliale, le bourgeon urétéral. Des interactions séquentielles et réciproques entre ces deux tissus sont indispensables au déroulement harmonieux des étapes ultérieures du développement. Le bourgeon urétéral se divise d'abord en forme de $T$, puis ses branches s'allongent et se divisent de façon dichotomique pour engendrer l'ensemble du système collec- teur (morphogenèse ramifiée). Le mésenchyme va être converti en épithélium et se différencier pour former les composants épithéliaux du néphron à l'exception des canaux collecteurs (Figure 1) [1].

La matrice extracellulaire est impliquée dans la morphogenèse rénale à trois titres. Elle constitue un substrat physique permettant l'organisation spatiale des cellules, contrôlant ainsi la morphogenèse ramifiée du bourgeon urétéral. Elle joue également un rôle plus
Inserm U489, Hôpital Tenon, 4 , rue de la Chine, 75970 Paris Cedex 20, France.

actif par sa fonction de réservoir des facteurs de croissance libérés par des protéases [2]. Elle interagit enfin avec des récepteurs membranaires, les intégrines, activant ainsi des voies de signalisation intracellulaire.

Le collagène de type XVIII est un collagène récemment identifié dans les membranes basales [3]. Sa structure laissait supposer qu'il pouvait être impliqué dans la morphogenèse puisqu'il possède un 
domaine similaire aux protéines frizzled auxquelles peuvent se lier les protéines de la famille Wnt dont plusieurs membres jouent un rôle important dans le développement rénal [1]. L'expression du collagène XVIII pendant le développement rénal et sa participation à la morphogenèse ramifiée viennent d'être élucidées dans un excellent article du groupe de Vainio [4]. Leurs résultats montrent que la localisation de ce collagène est corrélée avec le type de développement épithélial. Il est détecté à la première étape de l'organogenèse rénale et pulmonaire tout autour du bourgeon épithélial. Ultérieurement, son expression diffère suivant le type de mésenchyme. II est présent aux extrémités du bourgeon dans le poumon alors qu'il est observé autour de la tige du bourgeon urétéral dans le rein (Figure 2). Cette différence de localisation est corrélée avec le mode d'arborisation qui diffère dans les deux types de tissus. En effet, celle-ci est asymétrique dans le poumon alors qu'elle est dichotomique dans le rein (Figure 2). Grâce à des expériences de recombinaison associant en culture organotypique un bourgeon urétéral et un mésenchyme pulmonaire, le groupe de Vainio a montré que, contrairement au postulat établi jusqu'à présent, la morphogenèse ramifiée du bourgeon urétéral peut s'effectuer en présence d'un mésenchyme d'un tissu hétérologue tel que le poumon. Néanmoins, au premier stade de développement, les interactions du bourgeon urétéral, qui n'est pas encore divisé, avec le mésenchyme pulmonaire ne sont possibles qu'après un traitement du bourgeon par le GDNF (glial-derived nerve factor), facteur de croissance normalement produit par le mésenchyme rénal, qui confère au bourgeon urétéral la compétence de se diviser. Les autres signaux permettant l'arborisation du bourgeon urétéral proviennent du mésenchyme. En effet, en présence d'un mésenchyme pulmonaire, la morphogenèse ramifiée du bourgeon urétéral est de type «pulmonaire», c'est-à-dire asymétrique et non dichotomique, et s'ac- compagne d'une modification de la localisation du collagène XVIII qui devient identique à celle observée dans le poumon où il est présent aux extrémités du bourgeon épithélial. Ce collagène joue un rôle particulièrement important puisqu'il intervient directement dans les interactions inductives existant entre le mésenchyme pulmonaire et le bourgeon urétéral. $\varepsilon$ n effet, alors que les anticorps dirigés contre cette molécule ne modifient pas l'arborisation rénale en culture organotypique, ils réduisent le nombre de ramifications épithéliales dans le poumon et inhibent totalement la morphogenèse ramifiée du bourgeon urétéral lorsqu'il est cultivé avec le mésenchyme pulmonaire. Ces résultats suggèrent que le repositionnement $d u$ collagène XVIII est important pour sa fonction dans la morphogenèse pulmonaire. La diminution de l'expression du gène Wnt2 dans les cultures traitées par les anticorps anti-collagène XVIII et la présence d'un domaine frizzled suggèrent une interaction entre Wnt2 et le collagène XVIII.

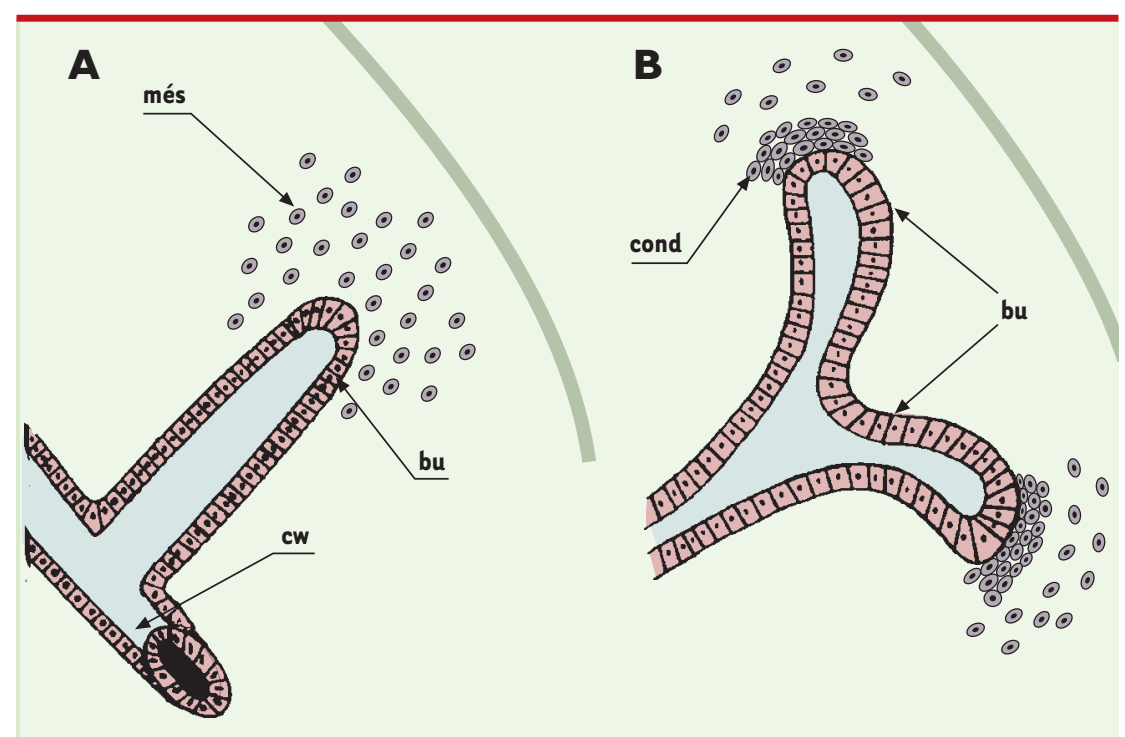

Figure 1. Représentation schématique des premières étapes du développement rénal. A. À 11 jours de gestation, le bourgeon urétéral (bu), formé à l'extrémité distale du canal de Wolff $(\mathrm{cw})$, envahit un petit domaine de mésenchyme dense, le blastème métanéphrique (més). B. Le bourgeon urétéral (bu) effectue un premier branchement en forme de T pendant qu'une partie du mésenchyme métanéphrique s'agrège à chacune de ses extrémités distales pour former des condensats (cond). 
Si le rôle du collagène XVIII n'est pas encore élucidé dans le développement rénal, en revanche, le rôle de l'endostatine, qui résulte d'un clivage protéolytique du domaine $\mathrm{NCl}$ du collagène XVIII, vient d'être récemment établi [7]. Dans un premier article, les groupes de Cantley et Sukhatme ont montré que cette protéine était capable de se fixer sur des cellules épithéliales tubulaires en se liant à des récepteurs de haute et de basse affinité, le glypican jouant le rôle de récepteur de basse affinité [8]. Les auteurs ont ensuite vérifié que l'endostatine était produite aux premiers stades de développement par le bourgeon urétéral. Elle est principalement exprimée dans la tige du bourgeon. $\varepsilon n$ culture organotypique, l'endostatine inhibe fortement la morphogenèse ramifiée du bourgeon en agissant à la fois sur les processus d'élongation et de division du bourgeon urétéral. À l'inverse, chez les souris déficientes en glypican-3, le récepteur de l'endostatine, on observe une hyperprolifération et une arborisation

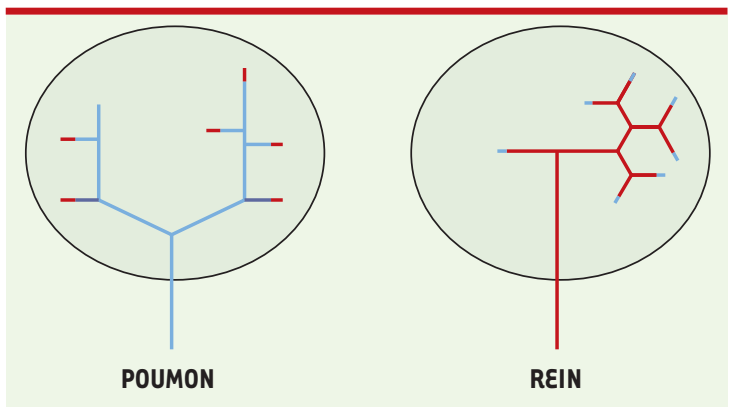

Figure 2. Représentations schématiques de l'arborisation et de la localisation du collagène XVIII dans le poumon et le rein. Poumon : le mode d'arborisation est asymétrique et le collagène XVIII en rouge est présent aux extrémités du bourgeon épithélial. Rein : I'arborisation du bourgeon urétéral est dichotomique. Le collagène XVIII en bleu est observé autour de la tige du bourgeon. accrue du bourgeon urétéral [9]. Ces résultats suggèrent que l'endostatine pourrait limiter le développement de branchements aberrants. Des mutations du glypican-3 ont été décrites chez les malades atteints de syndrome de Simpson-Golabi-Behmel, lié au chromosome $X$, et qui comporte, entre autres anomalies, une dysplasie rénale [10].

Ainsi, ces données récentes de la littérature ajoutent trois constituants de la matrice extracellulaire, le collagène XVIII, I'endostatine et le glypican-3 à la liste de plus en plus nombreuse des composants de la membrane basale qui participent de façon active à des processus aussi fondamentaux que la morphogenèse. $\nabla$
1. Lelongt B, Legallicier B, Ronco P. Bases moléculaires du développement rénal. Néphrologie 1998 ; 19 : 319-27.

2. Miner JH. Renal basement membrane components. Kidney Int 1999 ; 56 : 2016 24.

3. Saarela J, Rehn M, Oikarinen A, AutioHarmainen $\mathrm{H}$, Pihlajaniemi T. The short and long forms of type XVIII collagen show clear tissue specificities in their expression and location in basement membrane zones in humans. Am J Pathol 1998 ; 153:611-26.

4. Lin $Y$, Zhang $S$, Rehn $M$, et al. Induced repatterning of type XVIII collagen expression in ureter bud from kidney to lung type: association with sonic hedgehog and ectopic surfactant protein $C$. Development 2001; 128 : 1573-85.

5. Kispert A, Vainio S, Shen L, Rowitch DH, McMahon AP. Proteoglycans are required for maintenance of Wnt-11 expression in the ureter tips. Development 1996;
$122: 3627-37$.

6. Legallicier B, Trugnan G, Murphy $G$, Lelongt $B$, Ronco P. Expression of the type IV collagenase system during mouse kidney development and tubule segmentation. J Am Soc Nephrol 2001; 12 : 2358-69.

7. Karihaloo A, Karumanchi $S A$, Barasch J, et al. Endostatin regulates branching morphogenesis of renal epithelial cells and ureteric bud. Proc Natl Acad Sci USA 2001; 98 : 12509-14.

8. Karumanchi $S A$, Jha V, Ramchandran R, et al. Cell surface glypicans are lowaffinity endostatin receptors. Mol Cell 2001; 7 : 811-22.

9. Grisaru S, Cano-Gauci D, Tee J, Filmus J, Rosenblum ND. Glypican-3 modulates BMP- and FGF-mediated effects during renal branching morphogenesis. Dev Biol 2001 ; 231 : 31-46.

10. Pilia G, Hughes-Benzie RM, Mackenzie A, et al. Mutations in GPC3, a glypican gene, cause the Simpson-Golabi-Behmel overgrowth syndrome. Nat Genet 1996 ; 12 : 241-7. 